\title{
Biallelic variants in TSPOAP1, encoding the active-zone protein RIMBP1, cause autosomal recessive dystonia
}

\author{
Niccolò E. Mencacci, ${ }^{1,2}$ Marisa M. Brockmann, ${ }^{3}$ Jinye Dai, ${ }^{4}$ Sander Pajusalu, ${ }^{5,6,7}$ Burcu Atasu, ${ }^{8,9}$ Joaquin Campos, ${ }^{10}$ Gabriela Pino, ${ }^{10}$ \\ Paulina Gonzalez-Latapi, ${ }^{1}$ Christopher Patzke, ${ }^{4}$ Michael Schwake, ${ }^{1}$ Arianna Tucci, ${ }^{2}$ Alan Pittman, ${ }^{2}$ Javier Simon-Sanchez, ${ }^{8,9}$ \\ Gemma L. Carvill, ${ }^{1}$ Bettina Balint,, 2,11 Sarah Wiethoff, 2,9,12 Thomas T. Warner, ${ }^{2,13}$ Apostolos Papandreou, ${ }^{14,15}$ Audrey Soo, ${ }^{14,15}$ \\ Reet Rein, ${ }^{16}$ Liis Kadastik-Eerme, ${ }^{17,18}$ Sanna Puusepp, ${ }^{5,6}$ Karit Reinson, ${ }^{5,6}$ Tiiu Tomberg, ${ }^{19}$ Hasmet Hanagasi, ${ }^{20}$ Thomas Gasser, ${ }^{8,9}$ \\ Kailash P. Bhatia, ${ }^{2}$ Manju A. Kurian,, ${ }^{13,14}$ Ebba Lohmann, ${ }^{8,9}$ Katrin Õunap,, ${ }^{5,6}$ Christian Rosenmund, ${ }^{3}$ Thomas C. Südhof, ${ }^{4}$ \\ Nicholas W. Wood, ${ }^{2}$ Dimitri Krainc, ${ }^{1}$ and Claudio Acuna, ${ }^{40}$ \\ 1Ken and Ruth Davee Department of Neurology and Simpson Querrey Center for Neurogenetics, Northwestern University, Feinberg School of Medicine, Chicago, Illinois, USA. ${ }^{2}$ Department of Clinical and \\ Movement Neurosciences, UCL Queen Square Institute of Neurology, London, United Kingdom. ${ }^{3}$ Institute of Neurophysiology, Charité Universitätsmedizin, Berlin, Germany. ${ }^{4}$ Department of Cellular and \\ Molecular Physiology and Howard Hughes Medical Institute, Stanford University School of Medicine, Stanford, California, USA. ${ }^{5}$ Department of Clinical Genetics, Institute of Clinical Medicine, University \\ of Tartu, Tartu, Estonia. ${ }^{6}$ Department of Clinical Genetics, United Laboratories, Tartu University Hospital, Tartu, Estonia. ${ }^{7}$ Department of Genetics, Yale School of Medicine, New Haven, Connecticut, USA. \\ ${ }^{8}$ Cerman Center for Neurodegenerative Diseases (DZNE)-Tübingen, Tübingen, Germany. ${ }^{9}$ Center of Neurology, Department of Neurodegeneration, Hertie Institute for Clinical Brain Research, University of \\ Tübingen, Tübingen, Germany. ${ }^{10} \mathrm{Chica}$ and Heinz Schaller Foundation, Institute of Anatomy and Cell Biology, and ${ }^{11}$ Department of Neurology, Heidelberg University Hospital, Heidelberg, Germany. ${ }^{12 K}$ Klinik \\ für Neurologie mit Institut für Translationale Neurologie, Albert Schweitzer Campus 1, Gebäude A1, Münster, Germany. ${ }^{3}$ Reta Lila Weston Institute of Neurological Studies, UCL Queen Square Institute of \\ Neurology, London, United Kingdom. ${ }^{14}$ Molecular Neurosciences, Developmental Neurosciences, UCL Institute of Child Health, London, United Kingdom. ${ }^{15}$ Department of Neurology, Great Ormond Street \\ Hospital, London, United Kingdom. ${ }^{16}$ Children's Clinic, ${ }^{17}$ Department of Neurology and Neurosurgery, ${ }^{18}$ Neurology Clinic, and ${ }^{19}$ Radiology Clinic, Tartu University Hospital, Tartu, Estonia. ${ }^{20}$ Behavioural \\ Neurology and Movement Disorders Unit, Department of Neurology, Istanbul Faculty of Medicine, Istanbul University, Istanbul, Turkey.
}

Dystonia is a debilitating hyperkinetic movement disorder, which can be transmitted as a monogenic trait. Here, we describe homozygous frameshift, nonsense, and missense variants in TSPOAP1, which encodes the active-zone RIM-binding protein 1 (RIMBP1), as a genetic cause of autosomal recessive dystonia in 7 subjects from 3 unrelated families. Subjects carrying lossof-function variants presented with juvenile-onset progressive generalized dystonia, associated with intellectual disability and cerebellar atrophy. Conversely, subjects carrying a pathogenic missense variant (p.Cly1808Ser) presented with isolated adult-onset focal dystonia. In mice, complete loss of RIMBP1, known to reduce neurotransmission, led to motor abnormalities reminiscent of dystonia, decreased Purkinje cell dendritic arborization, and reduced numbers of cerebellar synapses. In vitro analysis of the $\mathrm{p}$.Cly1808Ser variant showed larger spike-evoked calcium transients and enhanced neurotransmission, suggesting that RIMBP1-linked dystonia can be caused by either reduced or enhanced rates of spike-evoked release in relevant neural networks. Our findings establish a direct link between dysfunction of the presynaptic active zone and dystonia and highlight the critical role played by well-balanced neurotransmission in motor control and disease pathogenesis.

\section{Introduction}

Dystonia is a disabling hyperkinetic movement disorder characterized by an excess of sustained, often repetitive, involuntary twisting movements, and abnormal postures (1). Dystonia, after Parkinson's disease and essential tremor, is the third most common movement disorder and has an estimated frequency of 732 per 100,000 in the general population (2). Clinically, dystonia can occur as an isolated symptom or in combination with other movement disorders or neurological abnormalities (3).

Authorship note: NEM and MMB are co-first authors. NWW, DK, and CA are co-senior authors.

Conflict of interest: The authors have declared that no conflict of interest exists. Copyright: @ 2021, American Society for Clinical Investigation.

Submitted: May 27, 2020; Accepted: February 3, 2021; Published: April 1, 2021

Reference information: J Clin Invest. 2021;131(7):e140625.

https://doi.org/10.1172/JCl140625.
Dystonia is thought to be a disorder of brain circuits involved in motor control $(4,5)$, with several lines of evidence locating its origin in the basal ganglia (6) or in the cerebellar connections (7). The precise cellular and molecular events responsible for the genesis of dystonic movements are not understood, hindering the development of more effective treatments. Importantly, pathogenic variants in a growing number of genes have been causally linked to dominant and recessive Mendelian forms of dystonia (8), allowing the development of genetic animal models and the initial delineation of converging molecular pathways in disease mechanisms (9). Along these lines, disruption of synaptic function has been recognized as a fundamental event in the pathogenesis of dystonia (10).

Synaptic function relies on fast and precise spike-triggered neurotransmitter release at the presynaptic active zone (11). The active zone is a cytomatrix composed of several large protein families, including Munc13s, RIMs, ELKs, $\alpha$-liprins, and RIM-binding proteins (RIMBPs) (12). These proteins contain multiple protein- 
protein interaction domains, which facilitates the formation of a densely interconnected protein network that enables docking and priming of synaptic vesicles for exocytosis and ensures rapid and efficient coupling between presynaptic calcium $\left(\mathrm{Ca}^{2+}\right)$ influx and synaptic vesicle fusion (13).

RIMBPs are central components of the active zone that, in conjunction with RIMs, determine the precise localization of presynaptic voltage-gated $\mathrm{Ca}^{2+}$ channels (VGCCs) and ensure tight coupling between presynaptic action potentials and synaptic vesicle exocytosis $(14,15)$. RIMBPs comprise 2 main brain isoforms, RIMBP1 and RIMBP2, which are structurally similar and consist of an SH3 domain in the N-terminal region, followed by 3 central fibronectin type-3 (FN3) domains, and 2 additional SH3 domains in their most distal C-terminal regions (16). Mechanistically, it is via their SH3 domains that RIMBPs bind to the proline-rich sequences in the cytosolic domain of presynaptic VGCCs, tethering them to the active zone $(14,17)$, and controlling the precision and fidelity of neurotransmitter release and synaptic transmission $(18,19)$.

Here, we demonstrate that rare homozygous truncating and missense variants in TSPOAP1 (MIM 610764, previously known as BZRAP1), the gene encoding RIMBP1, cause autosomal recessive dystonia in 3 unrelated families. In mice, deletion of RIMBP1 caused motor abnormalities reminiscent of dystonia as well as changes in the biochemical composition and morphology of the cerebellum. Interestingly, the pathogenic missense variant rendered abnormally large presynaptic $\mathrm{Ca}^{2+}$ transients and increased transmitter release in response to presynaptic firing. Altogether, our results highlight the critical role played by precisely fine-tuned synaptic transmission in normal motor function, and establish a direct link between RIMBP1-mediated neurotransmitter vesicle release and dystonia pathogenesis.

\section{Results}

Homozygous TSPOAP1/RIMBP1 variants cause autosomal recessive dystonia. Using a combination of homozygosity mapping and whole-exome sequencing (WES), homozygous variants in TSPOAP1 were independently identified as the top-candidate genetic cause of autosomal recessive dystonia in the 3 families reported herein (Figure 1 and Supplemental Tables 1 and 2; supplemental material available online with this article; https:// doi.org/10.1172/JCI140625DS1). The online platform GeneMatcher (20) was used to share results among the 3 groups that identified the variants.

Family A is a consanguineous pedigree of Gujarati Indian origin (parents of the affected subjects are first cousins) (Figure 1A). Homozygosity mapping revealed a single candidate chromosomal region corresponding to a large run of homozygosity $(\mathrm{ROH})$ of approximately 11.5 Mb on chromosome 17 (chr17: 53,415,850$64,831,657)$, which was unique to the 3 affected family members. After applying the filtering strategy to WES data, only a single candidate causative variant remained, a frameshift deletion in TSPOAP1 (NM_004758.3: c.538delG, p.Ala180Profs ${ }^{\star} 8$ ) located within the disease-associated $\mathrm{ROH}$ and shared by all 3 affected subjects. Sanger sequencing confirmed the presence of the variant and showed that both parents were heterozygous carriers, while the healthy sibling was homozygous for the reference allele, thus confirming complete disease cosegregation. This frameshift vari- ant is absent in more than 120,000 subjects listed in gnomAD, which contains more than 15,000 subjects of South Asian ancestry.

Family B is of Estonian origin and has been previously reported (21). There was no reported history of parental consanguinity. The proband is the only child in the family (Figure 1A). Chromosomal microarray analysis did not detect copy number variations. Homozygosity mapping revealed 3 large ROHs (chr4: 10,769,440-21,449,134; chr10: 54,942,171-63,972,930; chr17: $55,928,909-67,419,074)$ indicating likely unreported consanguinity. WES analysis identified a homozygous STOP-gain variant in TSPOAP1 (NM_004758.3: c.2449_2450delinsTG, p.Gln817*), located in the largest $\mathrm{ROH}$ as the best candidate. Sanger sequencing confirmed the presence of the homozygous variant in the proband and heterozygous in the mother (the father's DNA was not available). This variant is not present in gnomAD, which contains 2,418 Estonian subjects.

Family $\mathrm{C}$ is of Turkish origin (Figure 1A). There was no reported consanguinity in the family but the parents came from the same village. Three large ROHs (chr8: 33,406,218-42,407,799; chr11: 56,184,803-82,708,284; and chr17: 54,967,949-66,426,255) were found exclusively in the proband. WES analysis revealed 3 rare homozygous coding variants located within the identified ROHs: KCNU1 (NM_001031836: c.1280T>C, p.Ile427Thr [rs570237282]); NADSYN1 (NM_018161: c.278G>A, p.Arg93Gln [rs763061270]); and TSPOAP1 (NM_004758.3: c.5422G>A, p.Gly1808Ser [rs752560074]). Among these, only the variant in TSPOAP1 was homozygous also in the other siblings affected by dystonia. The variant is predicted to be pathogenic by all in silico prediction tools and has a combined annotation-dependent depletion score of 27.7. The variant is not present in the homozygous state in gnomAD (minor allele frequency 0.00001061) and is not present in the Great Middle East Variome database (22). Finally, the variant was absent in a further approximately 1000 Turkish families with unrelated medical conditions, thus excluding it as a common variant specific to the Turkish population.

TSPOAP1 variants identified in families $\mathrm{A}$ and $\mathrm{B}$ are both predicted to render truncated RIMBP1 products lacking most, if not all, functional domains. Thus, the carriers of these variants essentially represent RIMBP1-knockout (RIMBP1-KO) subjects (Figure 1D). In contrast, the p.Gly1808Ser variant found in family $\mathrm{C}$ is a missense variant located in the last RIMBP1 SH3 domain, which is critical for its binding to VGCCs $(14,17)$ and shows complete evolutionary conservation across species down to invertebrates and in the human homolog RIMBP2 (Figure 1D).

Loss-of-function variants are absent in approximately 15,000 in-house exomes sequenced at the 3 centers who analyzed the families and homozygous loss-of-function variants are not seen in a further approximately 130,000 subjects listed on gnomAD. Furthermore, we did not observe any homozygous, rare TSPOAP1 missense variant located within any of the 3 functionally relevant $\mathrm{SH} 3$ domains in both in-house exomes and gnomAD, indicating that homozygous variants in these regions are exceedingly rare and adding further evidence in support of the pathogenic role of the variant identified in family C. Finally, mining WES data from an additional approximately 700 cases affected with different forms of dystonia failed to identify additional cases with confirmed biallelic TSPOAP1 changes. 
A

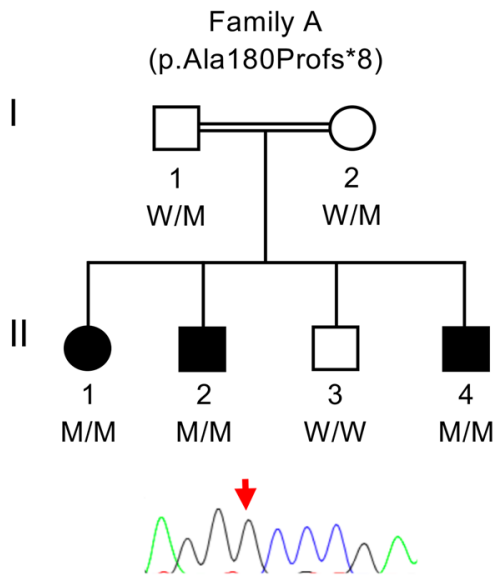

Reference

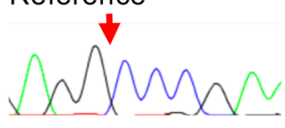

Homozygous

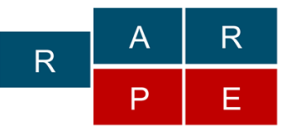

c.538delG
Family B

(p.GIn817*)
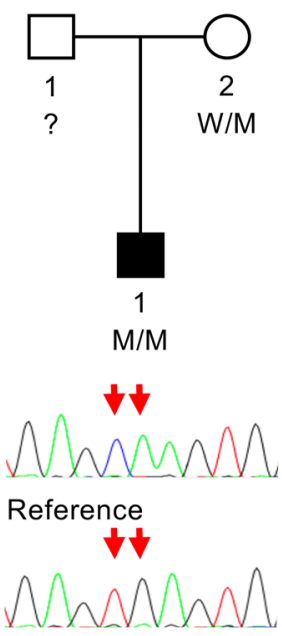

Homozygous

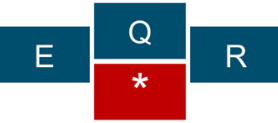

c.2449_2450delinsTG
Family C

(p.Gly1808Ser)

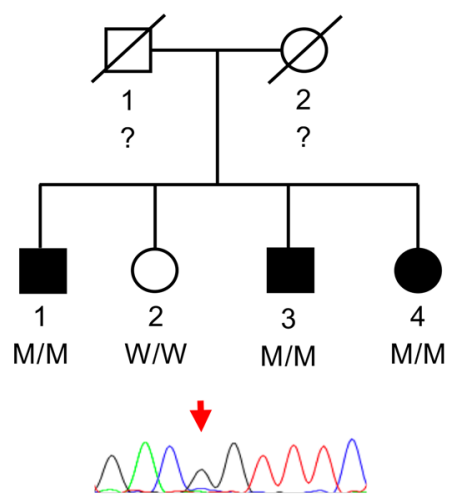

Reference

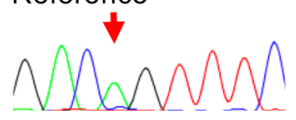

Homozygous

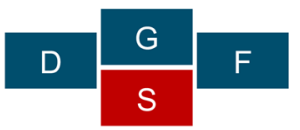

c. $5422 \mathrm{G}>\mathrm{A}$
B



C



Subject A-II.2

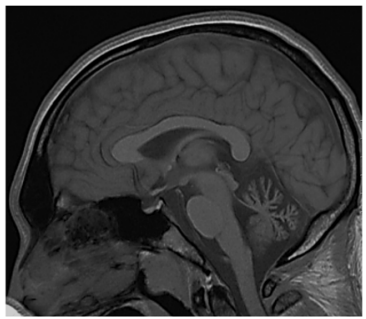

Subject A-II.4

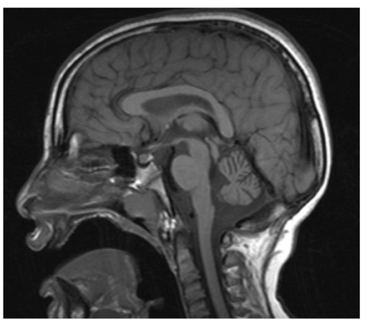

Subject B-II.1

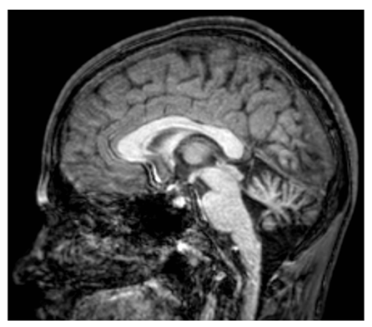

\section{D}

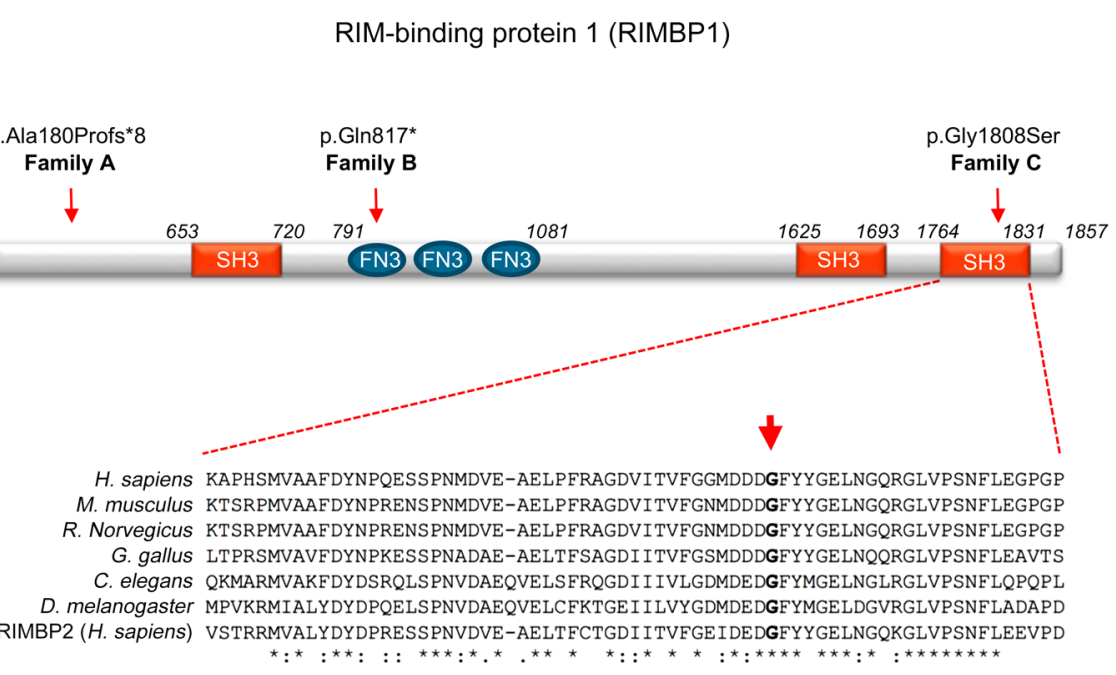


Figure 1. Pedigrees, genetic findings, and radiological features of subjects with pathogenic TSPOAP1/RIMBP1 variants. (A) Top: Pedigrees and variant status of affected (closed symbols) and healthy (open symbols) members of the 3 families with biallelic TSPOAP1 pathogenic variants. Bottom: Sanger sequencing validation of the variants and schematic representation of the variant effect on protein-coding sequence. W, wild-type alleles; $M$, mutant alleles. (B) Brain MRI sagittal T1-weighted images of subjects A-II.1 (age 28), A-II.2 (age 23), A-II.4 (age 13), and B-II.1 (age 17), demonstrating cerebellar atrophy with a predominance of the vermis in all 4 subjects carrying homozygous loss-of-function variants. (C) Cartoon illustrating the molecular interactions of RIM-binding proteins (RIMBPs) at the presynaptic active zone. RIMBPs bind with the first $\mathrm{SH} 3$ domain to Bassoon, with the FN3 domains to calcium-activated potassium channels (BK), and with the second and third SH3 domain to voltage-gated calcium-channels $\left(\mathrm{Ca}^{2+}\right)$ and RIM proteins. (D) Schematic representation of RIMBP1 structure including protein domains and localization of the identified variants. The amino acid residue Gly1808 is located in the C-terminal SH3 domain and shows complete evolutionary conservation across all species and in the human protein homolog RIMBP2. Asterisks indicate invariant residues (full conservation), whereas colons and periods represent strong and moderate similarities, respectively.

Clinical features of patients with biallelic TSPOAP1/RIMBP1 variants. The clinical features of all subjects with TSPOAP1 variants are summarized in Tables 1 and 2. In brief, all 4 subjects with homozygous loss-of-function TSPOAP1 variants (family A and B) shared a strikingly similar phenotype characterized by normal motor and language development, mild learning disabilities that first appeared in primary school, and onset in early teenage years of progressive generalized dystonia (Supplemental Video 1). Dystonia displayed a clear craniocaudal gradient in all 4 subjects, with dystonic movements severely affecting the craniocervical region and to a lesser extent the trunk and the 4 limbs. The impairment of the cranial muscles was particularly severe, resulting in prominent dysphonia and dysarthria and progressive difficulties with swallowing. Upper limb examination showed continuous dystonic posturing, muscle overflow, and writhing movements, resulting in impaired dexterity and severe handwriting difficulties. Gait examination showed progressive abnormalities including bilateral foot inward turning, tiptoeing, leg stiffening, and abnormal knee flexion, resulting in progressive loss of autonomous ambulation.

All 4 cases showed clear evidence of cognitive deterioration. Formal cognitive assessment was performed in case A-II.1 (age 27; extensive cognitive impairment) and in subject B-II.1 (age 10 and 17; progression from mild to moderate intellectual disability). Other additional neurological features that were only variably observed included generalized tonic-clonic seizures (subject A-II.4; EEG showed bilateral interictal epileptic activity), superimposed episodes of limb and painful truncal dystonia triggered by sudden movements and controlled by treatment with phenytoin (subject A-II.2), and lower limb spasticity with hyperactive reflexes (subject B-II.1).

Subjects from family $\mathrm{C}$ carrying the homozygous missense change p.Gly1808Ser presented with a milder dystonic phenotype, characterized by adult-onset focal dystonia or dystonic tremor (Supplemental Video 2). The index proband (C-II.4) developed dystonia at age 58 and on examination she had segmental dystonia affecting her neck and upper limbs. Two of the proband's siblings presented in their 60s with either isolated tremulous cer- vical dystonia and upper limb postural tremor (subject C-II.3) or isolated hand dystonic tremor (subject C-II.1).

Patients with homozygous TSPOAP1/RIMBP1 loss-of-function variants display progressive cerebellar atrophy. Brain MRI showed prominent cerebellar atrophy with a predominance for the vermis in all 4 subjects from families A and B (Figure 1B), a finding that is highly atypical in cases with generalized dystonia. Serial brain imaging in subject A-IV.2 (MRI at age 5, 8, and 14) and B-II.1 (MRI at age 15 and 17) documented progression of the cerebellar atrophy. The prominence of this radiological finding was in striking contrast with the paucity of classic clinical features of cerebellar dysfunction (i.e., ocular nystagmus, gait ataxia, limb dysmetria). Conversely, supratentorial, brainstem, and spinal cord parenchyma had an otherwise normal appearance and there were no signal abnormalities or volume loss in the basal ganglia. Brain imaging was reported as normal in the proband of family $\mathrm{C}$, but images were not available for direct review.

Brain expression profiles of TSPOAP1/RIMBP1 and its homo$\log$ RIMBP2 support an essential role for RIMBP1 in cerebellar Purkinje neurons $(5,7)$. Previous studies (16) as well as gene expression data sets from human and mouse brains, including GTEx (human brain expression) (23) and the Allen Brain Atlas (mouse brain expression) (24), showed that both TSPOAP1 and its homo$\log$ RIMBP2 are highly expressed across several brain regions in a partly overlapping and partly segregated manner (Supplemental Figure 1). Importantly, in the cerebellum the expression of TSPOAP1/RIMBP1 was very high, particularly in Purkinje cells, while the expression of RIMBP2 was low throughout. A similar pattern of expression was observed in the striatum as well, though less pronounced than in the cerebellum.

RIMBP1 and RIMBP2 are functionally redundant and can compensate for each other's function when individually deleted $(18,19)$. The particular RIMBP expression pattern in Purkinje cells indicates that compensation might not occur in these cells, and thus suggests Purkinje cells might be particularly susceptible to RIMBP1 dysfunction caused by the identified TSPOAP1 pathogenic variants.

Genetic deletion of RIMBP1, but not RIMBP2, triggers motor abnormalities in mice. To support the pathogenic role of the identified homozygous loss-of-function RIMBP1 variants, we pursued further studies in mice. For this, we generated RIMBP1-KO mice (Supplemental Figure 2A), as previously reported (25). RIMBP1KO mice displayed normal weight and no obvious developmental abnormalities (Supplemental Figure 2B), indicating that constitutive removal of RIMBP1 has no significant effects on mouse survival and gross development.

We then tested whether RIMBP1 KO impacts mouse motor behaviors that are commonly disrupted in dystonia models. First, quantification of open-field activity showed that RIMBP1-KO mice displayed an approximately $30 \%$ overall increase in spontaneous locomotor activity compared with WT littermates (Figure 2A). Second, in a beam-walk motor coordination test (Figure 2B), RIMBP1KO mice showed nearly $100 \%$ more slips and were significantly slower at crossing the beam than WT littermates (Supplemental Video 3). Third, suspension of mice by their tail (Figure 2C and Supplemental Video 4) for 10 or 20 seconds led to limb-clasping behavior in $80 \%$ of RIMBP1-KO mice and this percentage increased to $100 \%$ when the suspension lasted for 30 seconds (average clasp- 
Table 1. Clinical features of subjects from family A with homozygous pathogenic TSPOAP1 variants

\begin{tabular}{|c|c|c|c|}
\hline & A-II.1 & A-II.2 & A-II.4 \\
\hline Gender & Female & Male & Male \\
\hline Origin & Indian Gujarati & Indian Gujarati & Indian Gujarati \\
\hline Age at last examination (yr) & 31 & 26 & 20 \\
\hline TSPOAP1 variant ${ }^{A}$ & c.538delG, p.Ala180Profs*8 & c.538delG, p.Ala180Profs*8 & c.538delG, p.Ala180Profs*8 \\
\hline Motor development & Normal & Normal & Normal \\
\hline
\end{tabular}

Dystonia characteristics

\begin{tabular}{|c|c|c|c|}
\hline $\begin{array}{l}\text { Age at onset of motor } \\
\text { symptoms (yr) }\end{array}$ & 12 & 12 & 13 \\
\hline Symptoms at presentation & Loss of voice and speech difficulties & Speech difficulties & Rapid onset of speech difficulties \\
\hline $\begin{array}{l}\text { Symptoms of cranial, cervical, } \\
\text { laryngeal dystonia } \\
\text { (onset, yr) }\end{array}$ & $\begin{array}{c}\text { Severe orofacial involvement with } \\
\text { opening spasms, severe dysarthria and } \\
\text { dysphonia, dysphagia, severe torticollis } \\
\text { and retrocollis (12) }\end{array}$ & $\begin{array}{c}\text { Severe laryngeal dystonia (whispering } \\
\text { dysphonia), dysarthria (12) }\end{array}$ & $\begin{array}{l}\text { Anarthria and severe oral dystonia, } \\
\text { severe dysphagia, frequent spasms of } \\
\text { platysma muscle activated by } \\
\text { attempts to speak (13) }\end{array}$ \\
\hline $\begin{array}{l}\text { Upper limb involvement } \\
\text { (onset, yr) }\end{array}$ & $\begin{array}{l}\text { Continuous dystonic posturing and } \\
\text { movements worsened by action, muscle } \\
\text { overflow, impaired dexterity and } \\
\text { handwriting difficulties (17) }\end{array}$ & $\begin{array}{c}\text { Continuous dystonic posturing and } \\
\text { movements worsened by action, muscle } \\
\text { overflow, impaired dexterity and handwriting } \\
\text { difficulties (16) }\end{array}$ & $\begin{array}{l}\text { Continuous dystonic posturing and } \\
\text { movements worsened by action and with } \\
\text { muscle overflow, impaired dexterity and } \\
\text { handwriting difficulties (14) }\end{array}$ \\
\hline $\begin{array}{l}\text { Onset of lower limb } \\
\text { involvement (onset, yr) }\end{array}$ & $\begin{array}{l}\text { Tiptoe walking with extreme } \\
\text { knee flexion (17) }\end{array}$ & Bilateral foot inward turning (16) & Bilateral foot inward turning (16) \\
\hline Distribution at final examination & Generalized with craniocaudal gradient & Generalized with craniocaudal gradient & Generalized with craniocaudal gradient \\
\hline Gait & $\begin{array}{l}\text { Able to walk only with support; } \\
\text { severe dystonic features }\end{array}$ & $\begin{array}{l}\text { Possible only for short distances; } \\
\text { dystonic gait }\end{array}$ & $\begin{array}{l}\text { Possible only for short distances; bilateral } \\
\text { leg dystonic posturing, narrow-based }\end{array}$ \\
\hline Cognition & $\begin{array}{l}\text { Learning disability since school; } \\
\text { neuropsychology testing at age } 27 \text { revealed } \\
\text { extensive cognitive impairment }\end{array}$ & $\begin{array}{l}\text { Learning disability since school; cognitive } \\
\text { decline (not formally tested) }\end{array}$ & $\begin{array}{l}\text { Learning disability since school; cognitive } \\
\text { decline (not formally tested) }\end{array}$ \\
\hline Cerebellar signs & None & $\begin{array}{l}\text { Square wave jerks, slow and hypometric } \\
\text { saccades, mild intentional tremor and } \\
\text { dysmetria in } 4 \text { limbs, broad base }\end{array}$ & $\begin{array}{l}\text { Square wave jerks, slow and hypometric } \\
\text { saccades, mild dysdiadochokinesia in UEs }\end{array}$ \\
\hline Other neurological features & None & $\begin{array}{l}\text { Episodes triggered by sudden movements, } \\
\text { featuring painful truncal spasms, responsive } \\
\text { to AEDs but with no EEG correlate. }\end{array}$ & $\begin{array}{c}\text { Idiopathic generalized epilepsy with } \\
\text { generalized tonic-conic seizures with onset } \\
\text { at age } 4 \text {, resolved by age } 10\end{array}$ \\
\hline Brain MRI & Cerebellar atrophy & Cerebellar atrophy & Cerebellar atrophy \\
\hline Treatment trials & $\begin{array}{c}\text { BCF, TBZ, LD, BT (not effective). Currently on } \\
0.5 \mathrm{mg} \text { CNZ bid and } 2 \mathrm{mg} \text { THP od }\end{array}$ & $\begin{array}{l}\text { LD (no benefit). Currently on } 2 \mathrm{mg} \text { THP } \\
\text { (minimal improvement) and PHT }\end{array}$ & $\begin{array}{l}\text { THP, BT (not effective), } \\
\text { currently on CNZ }\end{array}$ \\
\hline Previous negative genetic testing & $\begin{array}{c}\text { TOR1A, THAP1, common mitochondrial DNA } \\
\text { mutations }\end{array}$ & $\begin{array}{c}\text { SCA1, SCA2, SCA3, SCA6, SCA7, SCA12, SCA17, } \\
\text { DRPLA, CSTB, FTXN, TOR1A }\end{array}$ & $\begin{array}{l}\text { TOR1A, GCH1, SGCE, ATM; normal } \\
\text { chromosomal microarray }\end{array}$ \\
\hline Previous investigations & $\begin{array}{c}\text { Normal copper and ceruloplasmin, ferritin, } \\
\text { acanthocytes, serum and urine amino and } \\
\text { organic acids, VLCFAs, blood smear, } \\
\text { phytanic and pristanic acid }\end{array}$ & $\begin{array}{l}\text { Normal white blood cell enzyme analysis, } \\
\text { purine metabolism, CSF (except marginal } \\
\text { 5-MTHF deficiency) }\end{array}$ & Normal EMG and NCS \\
\hline
\end{tabular}

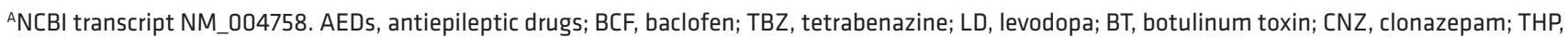
trihexyphenidyl; PHT, phenytoin; EMG, electromyography; NCS, nerve conduction studies; 5-MTHF, 5-methyltetrahydrofolate; VLCFAs, very long chain fatty acids; CSF, cerebrospinal fluid; UEs, upper extremities.

ing index of approximately $1.5 \pm 2.5$ ). In striking contrast, none of the WT mice exhibited hind limb clasping in any of these conditions (average clasping index approximately $0.5 \pm 0.1$; Figure 2C). Fourth, on rotarod testing, we did not detect significant differences between RIMBP1-KO and WT in their initial coordination nor in their learning rate (Supplemental Figure 2, C and D).

As human and mouse brains display high RIMBP1 and low RIMBP2 expression in the striatum and the cerebellum, we hypothesized that selective lack of RIMBP1, but not of RIMBP2, would lead to motor abnormalities. Consistent with this expectation, and in striking contrast with RIMBP1 KO, RIMBP2 KO neither affected the overall locomotor activity or beam-walk performance, nor caused limb-clasping behavior (Supplemental Figure 3, C-G). Thus, deletion of RIMBP1, but not RIMBP2, leads to motor abnormalities in mice.

Abnormal movements and postures in RIMBP1-KO mice upon acetylcholine muscarinic receptor stimulation. While RIMBP1-KO mice displayed several baseline motor abnormalities, we did not observe 


\section{Table 2. Clinical features of subjects from families B and C with homozygous pathogenic TSPOAP1 variants}

\begin{tabular}{|c|c|c|c|c|}
\hline & B-II.1 & C-II.1 & {$[-11.3$} & C-II.4 \\
\hline Gender & Male & Male & Male & Female \\
\hline Origin & Estonian & Turkish & Turkish & Turkish \\
\hline TSPOAP1 variant ${ }^{A}$ & c.2449_2450delinsTG, p.Gln817* & c.5422G>A, p.Gly1808Ser & c.5422G>A, p.Gly1808Ser & c.5422G>A, p.Gly1808Ser \\
\hline Motor development & Normal & Normal & Normal & Normal \\
\hline
\end{tabular}

Dystonia characteristics

\begin{tabular}{|c|c|c|c|c|}
\hline $\begin{array}{l}\text { Age at onset of motor } \\
\text { symptoms (yr) }\end{array}$ & 11 & $60 \mathrm{~s}$ & 67 & 58 \\
\hline $\begin{array}{l}\text { Symptoms of cranial, cervical, } \\
\text { laryngeal dystonia } \\
\text { (onset, yr) }\end{array}$ & $\begin{array}{l}\text { Mild dysarthria progressed into severe } \\
\text { dysarthria and orofacial-mandibular dystonia, } \\
\text { blepharospasm, retrocollis and laterocollis (14) }\end{array}$ & None & $\begin{array}{l}\text { Irregular dystonic horizontal } \\
\text { head tremor, mild right } \\
\text { torticollis }\end{array}$ & $\begin{array}{l}\text { Retrocollis, right laterocollis, } \\
\text { mild left torticollis, } \\
\text { right shoulder elevation }\end{array}$ \\
\hline $\begin{array}{l}\text { Upper limb involvement } \\
\text { (onset, yr) }\end{array}$ & $\begin{array}{l}\text { Continuous dystonic posturing and movements } \\
\text { worsened by action, muscle overflow, impaired } \\
\text { dexterity and handwriting difficulties (11) }\end{array}$ & $\begin{array}{c}\text { Dystonic hand resting } \\
\text { tremor }\end{array}$ & Bilateral postural tremor & $\begin{array}{l}\text { Mild bilateral writhing } \\
\text { movements }\end{array}$ \\
\hline $\begin{array}{l}\text { Onset of lower limb involvement } \\
\text { (onset, yr) }\end{array}$ & $\begin{array}{l}\text { Bilateral foot inward turning } \\
\text { (11) }\end{array}$ & None & None & None \\
\hline Distribution at final examination & Generalized with craniocaudal gradient & Focal & Focal & Focal \\
\hline Gait & $\begin{array}{l}\text { Possible only for short distances, very } \\
\text { unsteady, tiptoe walking, knee flexion and } \\
\text { severe dystonic posturing, narrow-based }\end{array}$ & Normal & Normal & Normal \\
\hline Other neurological features & Rigidity in right limbs & None & None & Bilateral Babinski sign \\
\hline Brain MRI & Cerebellar atrophy & NA & NA & Reported as normal (images NA) \\
\hline Treatment trials & $\begin{array}{c}\text { LD, CBZ (no benefit). Currently on LV } \\
\text { (some initial benefit), TBZ (some benefit), } \\
\text { BT (some benefit) }\end{array}$ & None & None & $\begin{array}{l}\text { THP, BCF, and BT injections } \\
\text { (beneficial) }\end{array}$ \\
\hline Previous negative genetic testing & TH, GCH1, HTT, АTP7B & None & None & HTT \\
\hline Previous investigations & $\begin{array}{l}\text { Normal serum and urine amino and organic } \\
\text { acids, MPS and sialic acid, purine/pyrimidines, } \\
\text { CSF (except diminished level of 5-MTHF). } \\
\text { Normal EMG and NCS. Muscle biopsy } \\
\text { (scattered angular atrophic muscle fibers } \\
\text { referring to denervated fibers) }\end{array}$ & None & None & Normal EMG and NCS \\
\hline
\end{tabular}

${ }^{A}$ NCBI transcript NM_004758. BCF, baclofen; TBZ, tetrabenazine; LD, levodopa; BT, botulinum toxin; THP, trihexyphenidyl; CBZ, carbamazepine; LV, leucovorin; EMG, electromyography; NCS, nerve conduction studies; 5-MTHF, 5-methyltetrahydrofolate; MPS, mucopolysaccharides; VLCFAs, very long chain fatty acids; CSF, cerebrospinal fluid; UEs, upper extremities; NA, not available.

any spontaneous abnormal movements or postures, the clinical hallmark of dystonia. Therefore, we challenged RIMBP1-KO mice with the nonselective muscarinic receptor agonist oxotremorine, which has been recently shown to promote overt dystonia-like abnormal movements and postures in another genetic mouse model of dystonia (26). RIMBP1-WT and -KO mice were injected with low doses of oxotremorine $(0.01 \mathrm{mg} / \mathrm{kg}$ intraperitoneally [i.p.]), and then the appearance of abnormal movements and postures was assessed for up to 1 hour after injection (Figure 2D).

RIMBP1-WT mice displayed mildly reduced spontaneous locomotion and rare events of head and body tremors or hind limb extension during the first 30 minutes after injection only. In striking contrast, littermate RIMBP1-KO mice showed prominent and prolonged abnormalities in postures and movements that lasted for at least 1 hour after oxotremorine injections (Figure 2D and Supplemental Video 5). These included a dramatic and sustained reduction in spontaneous locomotion, prolonged periods of immobility with hunched trunk postures associated with flexion of hind limbs and extension of forelimbs, slow and uncoordinated gait with extended body and abnormal hind limb postures, frequent excessive jerking of the head and body twitching, and prolonged motionless standing on hind limbs with uncoordinated movements of the forelimbs and occasional raising of a single paw (Supplemental Video 6). A quantitative analysis, using an established scale for motor abnormalities in mice (26), estimated that oxotremorine increased motor dysfunction in RIMBP1-KO mice 
A

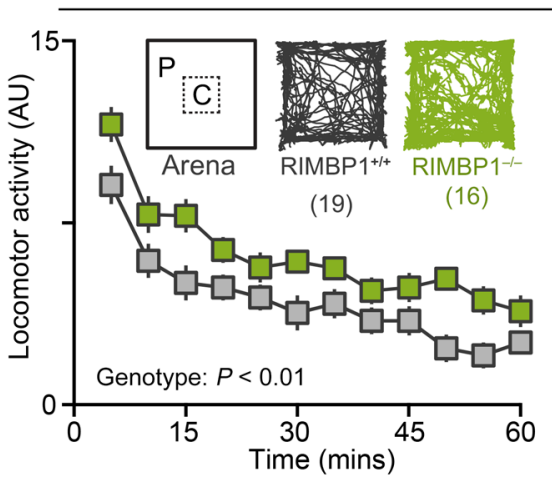

Locomotor activity



B

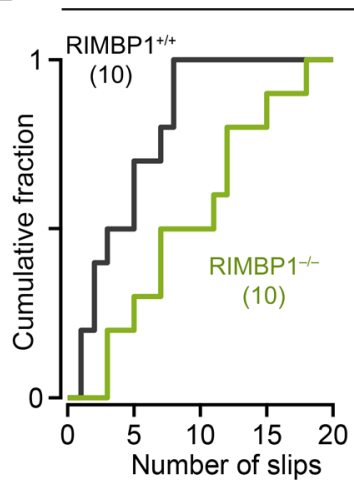

Beam walk


C

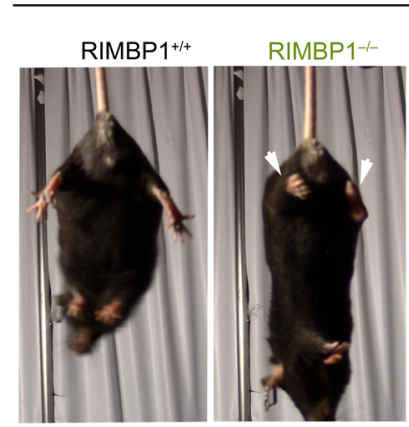

Limb clasping
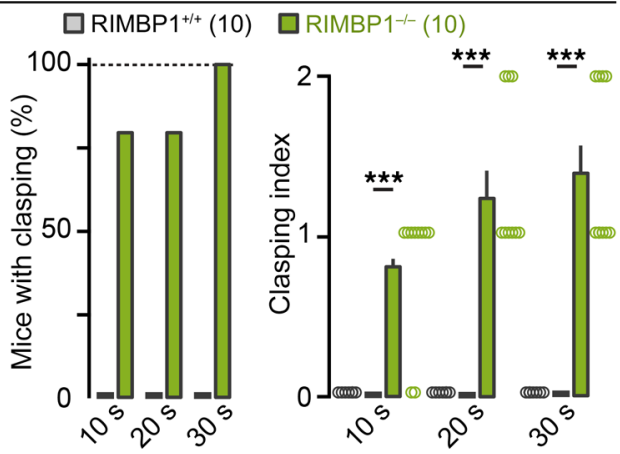

D


Figure 2. Motor abnormalities in RIMBP1-KO mice. (A) Open-field locomotor activity. Left: Locomotor activity as a function of time (bin width, 5 minutes). $P$ value by 1-way ANOVA. Middle: Total activity in RIMBP1-WT and RIMBP1-KO mice. Right: Comparison of relative activity in the periphery ( $P$, left) and the center ( $C$, right) of the open field arena in control and mutant mice. (B) Beam-walk test. Left: Cumulative distribution (left) and summary graphs (right) of total time to cross the beam in RIMBP1-WT and RIMBP1KO mice. Right: Cumulative distribution (left) and summary graphs (right) of total number of slips in RIMBP1-WT and RIMBP1 KO mice. (C) Limb-clasping test. Left: Representative pictures of a RIMBP1-WT (left) and a RIMBP1-KO (right) mouse during the tail suspension test used to measure limb clasping. Arrows highlight limb clasping in the RIMBP1-KO mouse. Left: Percentage of RIMBP1-WT and RIMBP1$\mathrm{KO}$ mice displaying limb clasping in tail suspension test lasting 10,20 , or 30 seconds. Right: Clasping index in control and mutant mice tail-suspended for 10,20 , or 30 seconds. (D) Abnormal movements and postures triggered by systemic oxotremorine. Top: Examples of abnormal postures (arrows) found in RIMBP1-KO mice after oxotremorine treatment (0.01 $\mathrm{mg} / \mathrm{kg}$ ). Bottom, left: Time course of abnormal motor scores after oxotremorine treatment $(0.01 \mathrm{mg} / \mathrm{kg})$. Motor scores were assessed in 2-minute bins. Abnormal motor score scale: 0 = normal motor behavior, $1=$ no impairment but slightly slowed movements, 2 = mild impairment with occasional abnormal postures and movements and ambulation with slow walk, 3 = moderate impairment with frequent abnormal postures and movements with limited ambulation, 4 = severe impairment with sustained abnormal postures without any ambulation or upright position. $P$ value by 1-way ANOVA. Bottom, right: Summed abnormal motor scores recorded for 60 minutes after drug injection. Data are presented as mean \pm SEM. Number of experiments: (A, right) $19 \mathrm{WT}, 16 \mathrm{KO}$; (B) $10 \mathrm{WT}, 10 \mathrm{KO}$; (C) $10 \mathrm{WT}, 10 \mathrm{KO}$; (D) $9 \mathrm{WT}, 10 \mathrm{KO} .{ }^{*} P$ $<0.05$; ${ }^{* *} P<0.001$ by 1 -way ANOVA or Student's $t$ test (in bar graphs in $\mathbf{A}-\mathbf{D}$ ). NS, not significant.

by approximately $300 \%$ compared with WT littermates (Figure 2D), indicating a severe susceptibility to muscarinic cholinergic stimulation caused by RIMBP1 deletion.

Cerebellar morphology and protein composition in mice lacking RIMBP1. Given the presence of progressive cerebellar atrophy in subjects with homozygous RIMBP1 truncation and the high expression levels of RIMBP1 in cerebellar Purkinje cells, we asked whether RIMBP1 deletion is causally linked to morphological or biochemical abnormalities in the mouse cerebellum. Gross visual inspection of cerebella from RIMBP1-WT and -KO mice did not show obvious size differences and quantitative analyses did not detect a significant difference in the cerebellar volume or the total number of Purkinje cells (Figure 3A). Furthermore, immunoblot- 
ting analysis of cerebellar lysates did not show significant changes in the levels of general neuronal markers between RIMBP1-WT and -KO mice (Supplemental Figure 3).

We found, however, that RIMBP1 KO led to significant morphological abnormalities in the dendritic arbors of Purkinje cells (Figure 3B). Quantification of the integrity of Purkinje cells, assessed by measuring the total area covered by calbindin immunolabeling in parasagittal cerebellar sections, revealed an approximately $30 \%$ loss of coverage in the more distal portion of Purkinje cell dendritic arbors (Figure 3B). Furthermore, the overall calbindin intensity was significantly decreased throughout the cerebellum $(31.6 \%$ reduction in the distal domain, $38.4 \%$ in the proximal domain; Figure $3 \mathrm{~B})$. This result was confirmed by the immunoblotting of cerebellar lysates, which showed an approximately $20 \%$ reduction in the calbindin signal in RIMBP1-KO mice (Figure 3D).

We also found that RIMBP1 deletion led to changes in the number of excitatory but not inhibitory synapses onto Purkinje cells. Purkinje cells receive 2 major excitatory inputs, parallel fibers and climbing fibers, which can be readily identified by expression of vesicular glutamate transporters vGluT1 and vGluT2, respectively. RIMBP1 deletion reduced both proximal and distal dendritic vGluT1 staining intensity by approximately $20 \%$ compared with WT controls (Figure 3C), whereas vGluT2-containing clusters were reduced by approximately $30 \%$ in the distal and by approximately $15 \%$ in the proximal dendritic arbors (Figure 3, D and E). Again, these results were confirmed by measurements of synapsin levels by immunoblotting of cerebellar lysates, which showed an approximately $20 \%$ reduction in RIMBP1-KO mice (Figure 3F; see complete unedited blots in the supplemental material). Immunoblotting analysis showed that neither the level of several activezone proteins (including RIM1 and RIMBP2) (Figure 3D, Supplemental Figure 4, and Supplemental Table 3) nor the levels of the SNAREs and associated proteins were significantly different in WT and KO cerebella. Likewise, the levels of VGCCs and BK channels (large conductance calcium-activated potassium channels) that interact with RIMBPs, as well as the levels of several postsynaptic proteins found in excitatory and inhibitory synapses, were not altered (Supplemental Figures 4 and 5).

Importantly, histological analysis of the cerebellum of 2-monthold RIMBP1-KO mice showed normal Purkinje cell dendritic trees and calbindin staining (Supplemental Figure 6, A-C), consistent with the idea that in mice, like in humans, cerebellar morphological abnormalities due to RIMBP1 mutations are progressive in nature. Finally, we also analyzed the structural integrity of the motor cortex and the striatum, 2 brain areas that play essential roles in dystonia pathogenesis. We found that neither the number of layer $\mathrm{V}$ pyramidal neurons in the motor cortex, nor the number of medium spiny neurons, cholinergic interneurons, or vGuT1 puncta signals in the striatum were changed in 6-month-old RIMBP1-KO mice (Supplemental Figure 6, D and E). Together, these results indicate that constitutive deletion of RIMBP1 in humans and mice impacts cerebellar but not striatal or cortical structural integrity.

Impact of the pathogenic RIMBP1 p.Gly1808Ser substitution on synaptic transmission. Subjects from families A and B described above carry TSPOAP1 truncating variants that essentially lead to the loss of all RIMBP1 functional domains. Therefore, these variants are expected to cause synaptic dysfunctions reminiscent of those observed in neurons with several RIMBPs knocked out simultaneously, including reduced priming, uncoupling of VGCCs from the active zone, and reduced synaptic transmission fidelity $(18,19,27,28)$.

Subjects from family C, in contrast, carry a missense variant (p.Gly1808Ser) that changes a single amino acid in the last SH3 domain of RIMBP1. Patients carrying this variant displayed a different dystonic phenotype, suggesting that the pathogenic mechanism triggered by this variant may be different from that of the truncating RIMBP1 variants. To address this, we used autaptic neuronal cultures (29), which allow for precise assessment of synaptic parameters (30) (Figure 4A). Because of the known functional redundancy between RIMBPs and RIMs (19), we performed all experiments in neuronal cultures prepared from RIMBP1, -2/ RIM1, -2 quadruple conditional KO (qKO) mice (19). These cultures were infected with lentivirus expressing Cre recombinase to delete all RIMBPs and RIMs, and then rescued with either the RIMBP control (RIMBP-WT) or RIMBP mutant (RIMBP-MUT) (Figure $4 \mathrm{~B}$; see complete unedited blots in the supplemental material), and analyzed via patch clamping or $\mathrm{Ca}^{2+}$ imaging (Figure 4, C and D).

Deletion of all RIMBPs and RIMs completely blocked spike-triggered glutamate release from cultured neurons (Figure 4, C and D), as previously described (19). Surprisingly, neurons rescued with RIMBP-MUT showed, on average, nearly 100\% larger excitatory postsynaptic current (EPSC) amplitude than RIMBPWT, indicating that the RIMBP1-p.Gly1808Ser variant does not compromise the function of RIMBP1 but rather causes abnormally increased synaptic transmission. As the pathogenic variant is located in the last SH3 domain of RIMBP1 (Figure 1D), which binds to VGCCs at the active zone and clusters them in close proximity of $\mathrm{Ca}^{2+}$ sensors (17), we hypothesized that p.Gly1808Ser may affect release by regulating the interaction with VGCCs, which in turn would impact total $\mathrm{Ca}^{2+}$ entry during action potential firing. Thus, we directly measured intraterminal $\mathrm{Ca}^{2+}$ levels using synaptically localized SynGCamp6F in qKO autaptic cultures rescued with either RIMBP-WT or -MUT (Figure 4, E-G). Neurons transduced with the RIMBP-WT rescue construct showed moderate presynaptic $\mathrm{Ca}^{2+}$ elevations in response to increasing numbers of action potentials (Figure 4, F and G). In striking contrast, neurons rescued with the pathogenic RIMBP-MUT variant displayed larger (by more than $100 \%$ ) $\mathrm{Ca}^{2+}$ transients (Figure 4G).

Finally, we studied whether p.Gly1808Ser affects the interaction of RIMBPs with VGCCs. We cotransfected HEK293T cells with a plasmid carrying an HA-tagged cytosolic C-terminal portion of the a1 subunits of P/Q (Cav2.1), known to mediate the interaction with RIMBPs, and a plasmid containing c-Myc-tagged RIMBP-WT or RIMBP-MUT (Figure 4H). Coimmunoprecipitation experiments revealed that RIMBP-MUT precipitated more than 3-fold higher amounts of Cav2.1 compared with RIMBP-WT (Figure 4, I and J; $P<0.03$ ). Altogether, these experiments indicate that p.Gly1808Ser causes abnormally increased neurotransmitter release via a mechanism that involves enhanced spike-triggered presynaptic $\mathrm{Ca}^{2+}$ entry, likely by recruiting a higher number of VGCCs at the release sites.

\section{Discussion}

We report here the identification of 3 pathogenic homozygous variants in TSPOAP1, the gene encoding the active-zone protein 
A
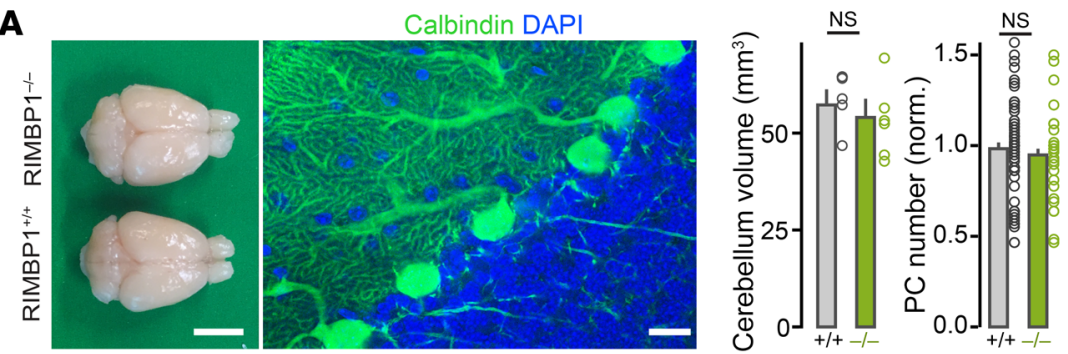

B


C
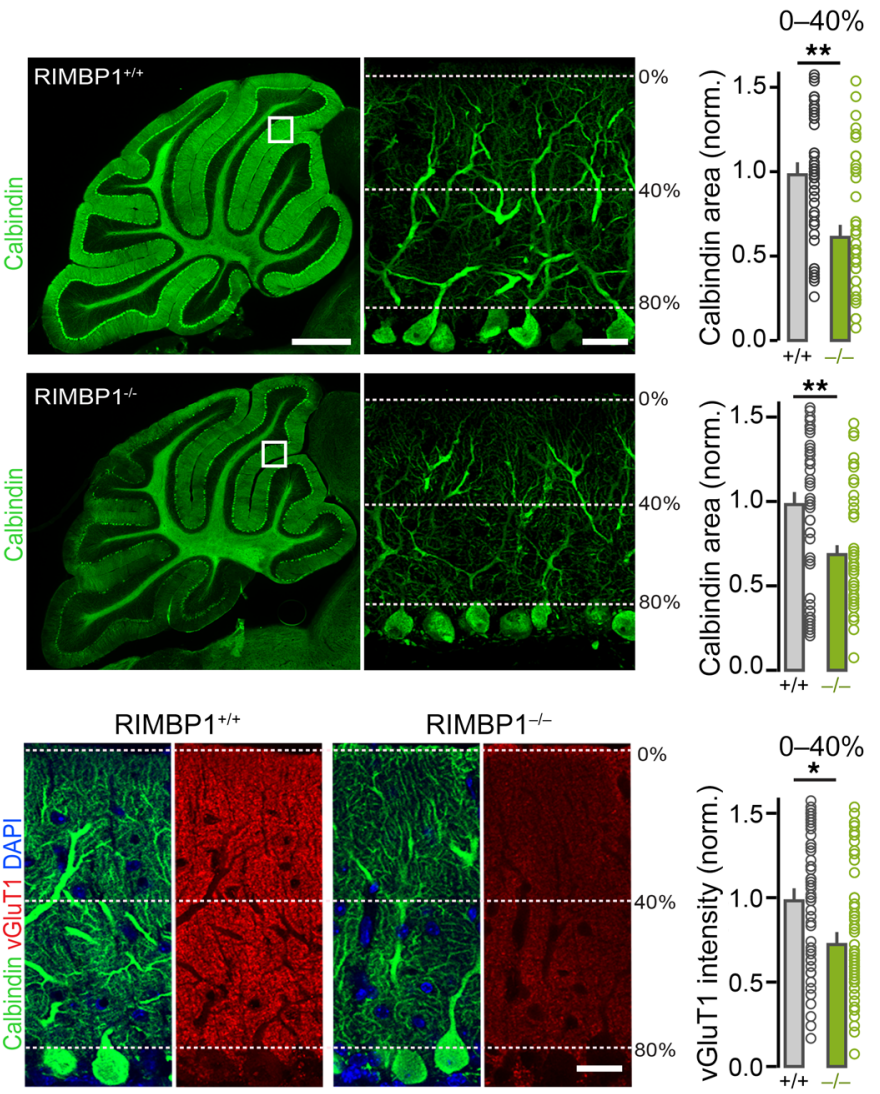

40-80\%
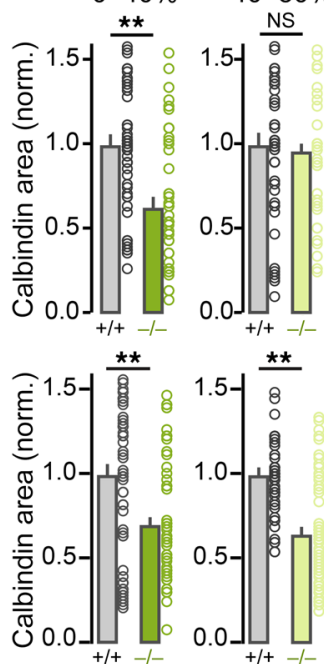

D

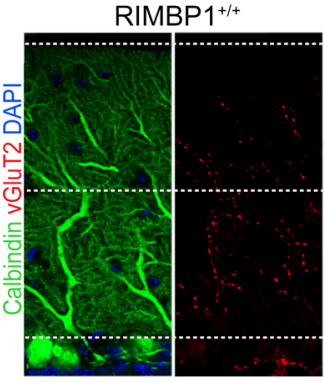

E

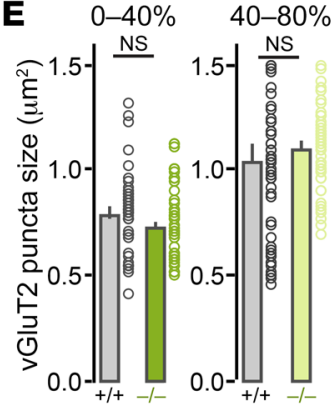

RIMBP $^{-/-}$



$F$





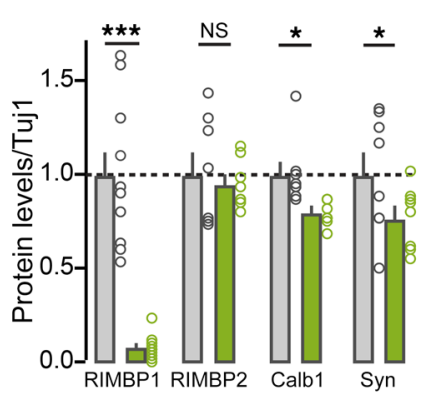

Figure 3. Cerebellar abnormalities in RIMBP1-KO mice. (A) Gross anatomy of the cerebellum. Left: Dorsal view of RIMBP1-WT and RIMBP1-KO brains. Middle: Confocal image of a parasagittal section stained with DAPI and anti-calbindin antibodies (green). Right: Summary plot of cerebellar volume (left) and of Purkinje cell (PC) number (right). Scale bars: $0.8 \mathrm{~cm}$ (left) and $20 \mu \mathrm{m}$ (right). (B) Structure of PC dendrites in RIMBP1 control and mutant mice. Left: Low-magnification images of parasagittal sections stained with anti-calbindin antibodies. Middle: High-magnification images (single optical sections) of the areas indicated with the white boxes on the left displaying outer $(0 \%-40 \%)$ and inner (40\%-80\%) PC dendritic domains. Right, top: Summary graphs of calbindin coverage (area) in different $\mathrm{PC}$ dendritic domains. Right, bottom: Summary graphs of calbindin intensity signal in the same PC dendritic domains. Scale bars: $1 \mathrm{~mm}$ (left) and $40 \mu \mathrm{m}$ (right). (C) Organization of parallel fiber synapses. Left: Representative confocal images of cerebellar sections from a RIMBP1-WT mouse. Middle: Representative images from a littermate RIMBP1-KO mouse. Sections were stained for calbindin (green, to label PCs), for vGluT1 (red, to visualize parallel fibers), and with DAPI (blue, cell nuclei). Right: Summary graphs of vGluT1 intensity for the outermost and innermost PC dendritic domains in RIMBP1-WT mice and -KO littermates. Scale bar: $40 \mu \mathrm{m}$. (D and E) Same as in C but for climbing fiber synapses stained with anti-vGluT2 antibodies. In this case, the density (D, right) and the size (E) of vGluT2-containing puncta were compared. Scale bar: $40 \mu \mathrm{m}$. (F) Levels of key proteins in cerebellar lysates. Left: Representative blots. Right: Summary plots of band intensity values normalized to Tuj1. Data are presented as the mean \pm SEM. ${ }^{*} P<0.05 ;{ }^{* *} P<0.01 ;{ }^{* *} P<0.001$ by Student's $t$ test. NS, not significant. Number of experiments (mice/sections): (A) Left: 5 WT, 6 KO; Right: 6/62 WT, 6/47 KO; (B) Intensity: 6/59 WT, 6/64 KO; Area: 6/57 WT, 6/60 KO; (C) vGluT1: 6/59 WT, 6/56 KO; (D and E) vGluT2: 6/54 WT, 6/60 KO; (F) $10 \mathrm{RIMBP}^{+/+}, 10 \mathrm{RIMBP}^{-/-}$.

RIMBP1, as a genetic cause of autosomal recessive dystonia. Two of these variants (p.Ala180 $\operatorname{Profs}^{*} 8$ and p.Gln817*) result in complete loss of RIMBP1 function either through early truncations and loss of all functional domains or through nonsense-mediated decay. The third variant (p.Gly1808Ser) is a missense variant located in the last SH3 domain of RIMBP1 and leads to abnormally increased synaptic transmission by likely recruiting more VGCCs to the active zone.

Clinically, the 4 subjects carrying homozygous truncating variants displayed a severe and progressive form of generalized dystonia featuring prominent craniocervical involvement with onset in early teenage years. Strikingly, in these patients dystonia was also 
A

Lentivirus infection

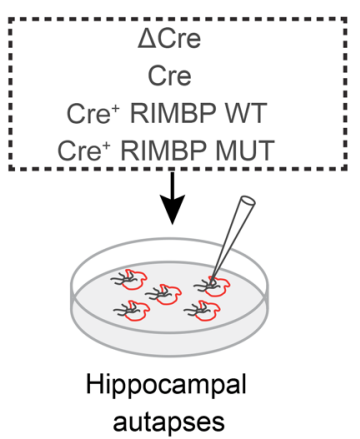

E

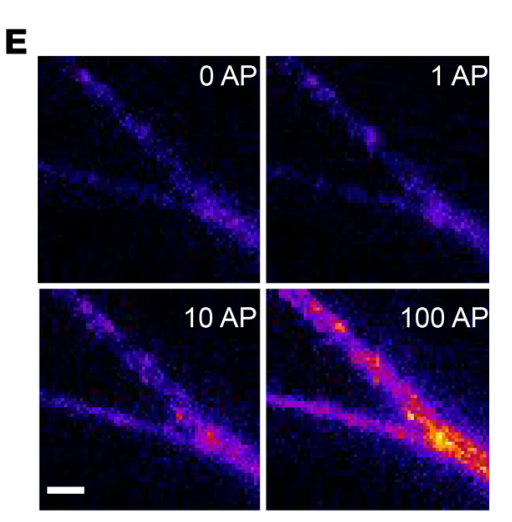

B



C



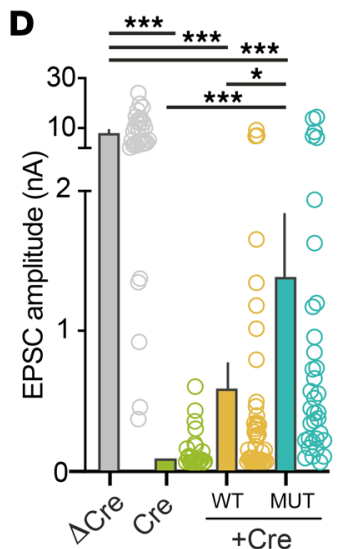

F



G

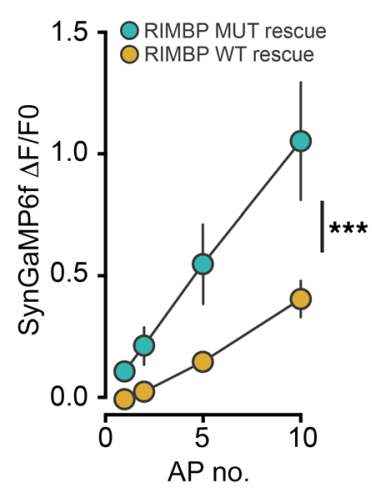

J

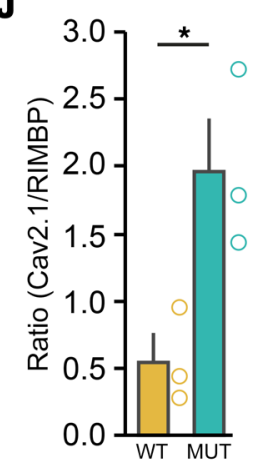

Figure 4. Impact of RIMBP1-p.Gly1808Ser variant on synaptic transmission. (A and B) Hippocampal autapses for assessing the role of p.Gly1808Ser on synaptic transmission in vitro. (A) Synaptic transmission in autapses was measured by whole-cell patch clamp recordings. Hippocampal autapses were prepared from RIMBP1, -2/RIM1, -2 quadruple conditional KO mice, and infected with lentiviruses expressing a recombinase-deficient version of Cre as control ( $\triangle \mathrm{Cre}$ ), Cre-recombinase (Cre), Cre+ RIMBP-WT, and Cre+ RIMBP-MUT constructs. (B) RIMBP levels in the presence of lentiviruses expressing $\Delta C$ re, Cre, Cre+ RIMBP-WT, and Cre+ RIMBP-MUT. In this experiment, $\alpha$-tubulin was used as a loading control. (C) Representative recordings of single action potential-evoked release (EPSC) in hippocampal autapses. (D) Summary graphs of EPSC amplitudes rescued with either RIMBP-WT or with the pathogenic RIMBP-MUT construct. (E-C) Direct measurements of spike-triggered presynaptic calcium entry. (E) Fluorescence images of a representative hippocampal dendrite expressing SynCCaMP6f under basal conditions (O AP), or after 1, 10, and 100 action potentials (APs). Scale bar: $2.5 \mu \mathrm{m}$. (F) Time course of presynaptic fluorescence signals ( $\triangle \mathrm{F} / \mathrm{F} 0$ ) as a function of time following 1, 2, 5, or $10 \mathrm{APs}$. (G) Summary graph of presynaptic fluorescence signals for different AP frequencies in autaptic cultures infected with viruses expressing Cre ${ }^{+}$RIMBP-WT and Cre+ RIMBP-MUT. (H-J) RIMBP pathogenic missense variant impacts RIMBP-Ca ${ }^{2+}$-channel interaction. (H) Experimental strategy for assessing the interaction of RIMBP with calcium channels using coimmunoprecipitations (co-IPs). (I) Cell lysates from HEK293T cells expressing CaV2.1-HA and RIMBP-WT-Myc, or CaV2.1-HA and RIMBP-MUT-Myc were immunoprecipitated with anti-Myc antibodies. Input fractions (left, $1 \%$ of total) and IPs were analyzed by immunoblotting with antibodies against the following: Myc epitope (RIMBP), HA epitope (CaV2.1), and GAPDH as a negative control. (J) Summary graph of 3 coimmunoprecipitation experiments performed as in I. Data are presented as the mean \pm SEM. ${ }^{*} P<0.05 ;{ }^{* *} P<0.001$ by 1 -way ANOVA with Tukey's post hoc analysis (D and $\left.\mathbf{G}\right)$ or Student's $t$ test (J). NS, not significant. Number of experiments (cells/cultures): (C and D) $\Delta$ Cre (32/3), Cre (24/3), RIMBP-WT (43/3), RIMBP-MUT (35/3); (F and G) Cre+ RIMBP-WT (17/3), Cre+ RIMBP-MUT (16/3); (J) RIMBP-WT (3), Cre+ RIMBP-MUT (3). 
associated with paucisymptomatic progressive cerebellar atrophy, a presentation that to date has been described in only a small number of families (31). On the other hand, the 3 subjects carrying the missense variant p.Gly1808Ser showed a milder phenotype featuring later-onset focal dystonia, without clear evidence of significant cerebellar structural defects.

Like in humans, complete removal of RIMBP1 in mice resulted in several motor abnormalities commonly observed across different genetic mouse models of dystonia (32). These included dramatically abnormal movements and postures in response to subthreshold injections of the muscarinic agonist oxotremorine, known to unmask the appearance of dystonia-like movements in other genetic models of dystonia (26). Loss of RIMBP1 in mice also resulted in structural abnormalities in the cerebellum, including shortening of the dendritic tree of Purkinje cells, reduced excitatory synaptic puncta, and abnormal cerebellar expression of synaptic markers. Importantly, we found that such histological abnormalities were not present in younger (2-month-old) RIMBP1-KO mice, indicating that the loss of RIMBP1 function leads to progressive structural abnormalities in the cerebellum, more remarkably in cerebellar Purkinje cells. The lack of clinical signs of cerebellar dysfunction despite the presence of prominent cerebellar atrophy in subjects with homozygous TSPOAP1/RIMBP1 truncating variants is puzzling. We speculate that, given its progressive nature, the degree of cerebellar dysfunction may still be too mild to cause prominent signs of cerebellar dysfunction in patients that lack RIMBP1, yet severe enough to cause subtle synaptic transmission abnormalities in cerebellar circuits and in other brain regions, like the striatum or cerebral cortex, resulting in the production of dystonic movements.

Our results indicate that RIMBP1, but not RIMBP2, is a key molecular link between synaptic dysfunction and dystonia. This is likely because in mice and humans RIMBP1 is heavily expressed in cerebellar Purkinje cells, where the homologous and functionally redundant RIMBP2 is virtually not expressed, thus preventing functional compensation and making Purkinje cells particularly susceptible to the effect of genetic variants affecting RIMBP1 function. The association of cerebellar structural abnormalities and dystonia observed in both humans and mice lacking RIMBP1 further supports the increasingly recognized role of cerebellar dysfunction in dystonia pathogenesis (7). Recent work in mice showed that abnormal and irregular burst firing of Purkinje cells, as well as in neurons in the deep cerebellar nuclei, can be observed in several murine models of dystonia (33-37). Furthermore, impaired cerebellar output may directly cause dystonia by altering basal ganglia activity and plasticity of corticostriatal pathways (38). In this context, given the essential role of RIMBPs in regulating precise and reliable synaptic release (18), we speculate that RIMBP1 deletion may cause irregular synaptic transmission of Purkinje cells, resulting in aberrant plasticity at corticostriatal synapses. This effect is likely further precipitated by oxotremorine, as overactivation of cholinergic M1 receptors on striatal neurons has been shown to have a strong effect on disrupting corticostriatal synaptic plasticity (39).

It is worth emphasizing that our work does not establish a causal link between the observed cerebellar structural abnormalities and dystonia pathogenesis in patients and mice with complete RIMBP1 loss of function. Indeed, RIMBP1 is also highly expressed in the motor cortex and in the striatum, 2 brain structures with an established role in dystonia pathophysiology. Our initial histological analysis of these 2 regions did not identify major differences in cell numbers or morphology between RIMBP1-WT and -KO mice. However, a detailed electrophysiological characterization of cerebellar and corticostriatal synapses will be necessary to further understand the functional neuroanatomy of this condition. Along these lines, another compelling hypothesis is that RIMBP1 deletion may affect the activity of olivocerebellar excitatory synapses, the disruption of which has been recently shown to be able to cause dystonic movements in mice (40). Intriguingly, we observed in the cerebella of RIMBP1-KO mice a reduced number of vGluT2 puncta, a marker of the cerebellar climbing fibers originating from the inferior olives, hence suggesting a possible critical role of this structure. Overall, circuit-specific deletions of RIMBP1, combined with behavioral analysis, will shed crucial insight into the contribution of subtle synaptic abnormalities in these brain structures to the pathogenesis of RIMBP1-related dystonia.

RIMBPs are enriched at the interface between the active-zone cytomatrix and the presynaptic membrane where they directly interact with RIMs and P- and Q-type VGCCs via their SH3 domains. Via these interactions, RIMBPs promote accumulation of VGCCs (i.e., Cav2.1 and Cav2.2) at the release sites, and thereby regulate the dynamics of transmitter release in response to presynaptic action potentials. Therefore, mechanistically, RIMBP1 variants can influence synaptic function by altering the clustering of release-relevant presynaptic VGCCs. Loss-of-function variants are expected to reduce the density of active-zone VGCCs, as observed in central synapses of mice lacking RIMBPs $(18,19,28,41)$. Conversely, the missense variant found in family C (p.Gly1808Ser) increased the extent of transmitter in response to action potentials. We also observed that this variant increased presynaptic $\mathrm{Ca}^{2+}$ elevation per action potential and enhanced spike-triggered intraterminal $\mathrm{Ca}^{2+}$, likely due to an increased number of VGCCs per active zone. Together, these results indicate that both decreased or increased density of VGCCs at the active zone is key in dystonia pathogenesis.

RIMBPs can also interact with presynaptic $\mathrm{Ca}^{2+}$-activated

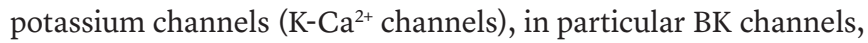
via their central FN3-type domains and regulate their clustering at the release sites (42). Although the exact physiological significance of this interaction remains to be determined, it is likely that active-zone BK channels limit the extent of membrane depolarization and transmitter release during sustained trains of presynaptic action potentials. Therefore, RIMBP1 variants may also alter the density of BK channels. Although our results indicate that the total amount of BK alpha subunit was not altered in RIMBP1-KO mice, it is not known if the nanoscale organization of BK channels might be impaired both in Purkinje cells as well as in other brain regions involved in dystonia pathogenesis. At the cellular level, $\mathrm{K}^{-\mathrm{Ca}^{2+}}$ channel dysfunction leads to uncoordinated firing patterns in Purkinje cells, which is expected to alter the fidelity and reliability of information transfer to downstream targets in the deep cerebellar nuclei (43). This phenotype is identical to that observed upon genetic removal of RIMBPs from other central synapses (41).

Importantly, evidence from human genetic studies supports the role of perturbed presynaptic neurotransmitter release in the pathogenesis of hyperkinetic movement disorders. Pathogenic dominant variants in the RIMBP1 binding partners CACNA1A and 
KCNMA1, encoding Cav2.1 and BK channels, respectively, have been linked to a variety of human movement disorders, including episodic ataxia (44) and paroxysmal dyskinesias $(45,46)$. Moreover, recessive variants in CACNA1A, CACNA1B, and KCNMA1 cause progressive epileptic encephalopathy variably associated with cerebellar atrophy $(47,48)$ or dyskinesias $(49)$. Furthermore, the protein product of PNKD, another gene linked to a paroxysmal dyskinetic movement disorder, has been recently shown to localize at synapses where it interacts with RIM1 and RIM2, modulating neurotransmitter release (50). Finally, a gain-of-function missense variant in the presynaptic protein MUNC13A, causally linked to a complex dyskinetic movement disorder, was recently shown to cause pathologically enhanced neurotransmitter release (51), a synaptic phenotype similar to what we observed for the RIMBP1 variant p.Gly1808Ser.

In conclusion, our results show that RIMBP1 dysfunction causes dystonia in humans and abnormal movements and postures in mice. To date, TSPOAP1/RIMBP1 haploinsufficiency had only been genetically linked to increased risk of autism (52). Our results establish a causal link between dysfunction of the active-zone machinery and dystonia in subjects with biallelic TSPOAP1/RIMBP1 genetic defects, which suggests a potential broader role for presynaptic dysfunction in dystonia pathogenesis. As RIMBP1 dysfunction leads to unreliable synaptic transmission, we hypothesize that restoring the precision of synaptic release pharmacologically may represent a promising therapeutic angle to treat dystonia and other hyperkinetic movement disorders. The RIMBP1-KO mouse model we developed could be instrumental not only to test this hypothesis but also to disentangle the circuit abnormalities underlying the pathogenesis of RIMBP1-related dystonia and to further investigate the complex interaction between the cerebellum and basal ganglia.

\section{Methods}

\section{Patients}

Gene mapping. DNA was extracted from peripheral lymphocytes following standard protocols. A genome-wide analysis genotyping scan was performed in all 6 members of family A and in the proband of family B using the HumanCytoSNP-12 DNA Analysis BeadChip Kit (Illumina), according to the manufacturer's instructions. Homozygosity mapping was performed using PLINK v.1.079 (53). In family $\mathrm{C}$, homozygosity mapping was performed using WES data analyzed with H3M2 software (54).

WES. WES was performed in all 3 affected children and both parents for family A, in the proband only for family $\mathrm{B}$, and in the proband and her unaffected sibling for family C. WES and bioinformatic analysis were performed as previously described (55-57). Given the reported history of parental consanguinity in family A and the result of homozygosity mapping suggesting cryptic parental relatedness in families B and C, the analysis focused on homozygous coding and essential splice-site variants. Variants were not considered if they had a read depth of less than 5 and a minor allele frequency (MAF) of greater than 0.005 in Exome Aggregation Consortium, Complete Genomics 69, 1000 Genomes project, and Exome Variant Server, as well as in in-house databases containing WES data from healthy controls or subjects with unrelated disorders. The summary of WES quality metrics for the families included in this study and full list of genetic variants remaining after the initial filtering strategy are listed in Supplemental Tables 1 and 2.

\section{Mice}

General. We used the following mouse lines: RIMBP1-KO, RIMBP2KO, and RIM1, -2/RIMBP1, -2 qKO. Mice were generated and maintained as described previously $(18,19)$.

Measurements of motor performance. All behavioral tests were performed in 6-month-old mutant and control littermate male mice between 1 and 7 pm. (i) Open field locomotion. Mice were video tracked in an open field arena for 60 minutes. Distance traveled, speed, and overall activity were analyzed using Viewer software (BIOBSERVE). (ii) Limb clasping. Mice were suspended by their tails for 10,20 , or 30 seconds and the percentage of animals displaying clasping behavior at each time point was measured. To quantify limb clasping, we used the following "clasping index": 0 = no clasping, $1=$ single limb clasping, and 2 =clasping in 2 or more limbs. In all experiments, mice were videotaped and analysis of video recordings was performed offline in a blinded manner. (iii) Beam-walk test. Mice were trained to cross a suspended beam over 3 consecutive days and on the fourth day they were videotaped and the time that took for them to cross the beam (10\%-90\% of total distance) as well as the total number of slips during the crossing, was quantified. (iv) Accelerating rotarod. We used a 5-station rotarod treadmill (ENV-575M, Med Associates) equipped with an 8-80 rpm rate of acceleration over 300 seconds. Time and speed to fall off was quantified automatically (Med Associates). (v) Motor behavior after systemic injections of oxotremorine. WT and littermate RIMBP1-KO male mice were habituated in a clear plastic cage for 10 minutes and then treated with an i.p. injection of $0.01 \mathrm{mg} / \mathrm{kg}$ oxotremorine methiodide (Sigma-Aldrich) dissolved in normal saline solution $(0.9 \% \mathrm{NaCl})$. Mice were videotaped for 60 minutes and motor behavior assessed every 10 minutes using a previously proposed scale for quantification of abnormal movements and postures (26). Two observers blinded to the genotype analyzed the experiment and rated the motor behavior of the mice.

Morphological measurements and synaptic density. All morphological measurements were performed in 6-month-old mice, except for those shown in Supplemental Figure 6, A-C, which were done in 2-month-old mice. To obtain a quantitative estimation of cerebellar size, we transversally cut $(50 \mu \mathrm{m})$ paraformaldehyde-fixed cerebella, stained them with DAPI, imaged them under epifluorescence microscopy, and reconstructed them in 3D using Image (NIH). To assess Purkinje cell number, 40- $\mathrm{m}$ parasagittal sections from either RIMBP1-WT or RIMBP1-KO mice were immunostained with anti-calbindin antibodies, imaged using confocal microscopy, and analyzed with NIS-Elements AR software (Nikon), comparing the density of Purkinje cells by counting their somas. To measure excitatory synapse density, the cerebella of 6 RIMBP1-WT and 6 RIMBP1-KO mice were cut parasagittally $(30 \mu \mathrm{m})$ and stained with antibodies against calbindin (1:4000, mouse; Sigma-Aldrich) to label Purkinje cell dendritic profiles, and either vGluT2 (1:500, guinea pig; Millipore) or vGluT1 (1:1000, guinea pig; Millipore) antibodies to label climbing and parallel fiber input, respectively. To measure inhibitory synapse density on Purkinje cells, 4 RIMBP1-WT and 4 RIMBP1-KO cerebella were cut parasagittally $(30 \mu \mathrm{m})$ and stained with anti-GAD65 (1:1000, mouse; DSHB) and anti-calbindin (1:1000, rabbit; Millipore) antibodies. To measure morphological parameters in the striatum and motor cortex, 
the forebrain of RIMBP1-WT and RIMBP1-KO mice was cut transversally $(30 \mu \mathrm{m})$ and stained with antibodies against DARPP-32 (1:4000, rabbit; Cell Signaling Technology), ChAT (1:1000, mouse; SigmaAldrich), NeuN (1:1000, mouse; Merck Millipore), and vGluT1 (1:1000, rabbit, custom-made). Synaptic puncta were imaged using a Nikon confocal microscope (A1RSi+) controlled by NIS-Elements AR software. All the acquisition parameters were kept constant between WT and mutant conditions. A complete list of antibodies and their clone numbers is shown in Supplemental Table 3.

Neuronal cultures, electrophysiology, and calcium imaging. Autaptic cultures from RIM1, -2/RIMBP1, -2 qKO mice were prepared at P1 (19, 30). Briefly, neurons were plated on a sparse layer of glial microislands under growth-permissive substrate to obtain single-neuron microislands. Twenty-four to 36 hours after plating, cultures were infected with either Cre-expressing or a recombination-deficient $\Delta$ Cre-expressing vector as a control. For rescue experiments, neurons were additionally infected (24-36 hours after plating) with either the RIMBP-WT or RIMBP-MUT construct carrying the pathogenic p.G1808S variant. All autaptic cultures were grown for at least 2 weeks before recordings. Patch-clamp electrophysiology was performed at DIV 14-18 using an electrode filled with an intracellular solution containing (in $\mathrm{mM}$ ): 136 $\mathrm{KCl}, 17.8$ HEPES, 1 EGTA, $4.6 \mathrm{MgCl}_{2}, 4 \mathrm{Na}_{2} \mathrm{ATP}, 0.3 \mathrm{Na}_{2} \mathrm{GTP}$, and 12 creatine phosphate, and $50 \mathrm{U} / \mathrm{mL}$ phosphocreatine kinase (approximately 300 mOsm; pH 7.4). The extracellular solution contained (in $\mathrm{mM}$ ): $140 \mathrm{NaCl}, 2.4 \mathrm{KCl}, 10$ HEPES, $2 \mathrm{CaCl}_{2}, 4 \mathrm{MgCl}_{2}$, and 10 glucose ( $\mathrm{pH}$ adjusted to 7.3 with $\mathrm{NaOH}$, approximately 300 mOsm). Single action potentials were evoked via a 2-ms depolarization pulse (from -80 to $0 \mathrm{mV}$ ) in voltage clamp while simultaneously recording evoked currents at $-80 \mathrm{mV}$ holding potential (30). For presynaptic $\mathrm{Ca}^{2+}$ imaging, autaptic cultures were additionally infected at DIV 1 with a lentivirus expressing SynGCamp6. To evoke action potentials, neurons were patch clamped as indicated above and then spikes were triggered by direct current injection in current clamp. Images in $\mathrm{Ca}^{2+}$ imaging experiments were acquired using a 490-nm LED system (pE2; CoolLED) at a $20 \mathrm{~Hz}$ sampling rate with a $50 \mathrm{~ms}$ exposure time.

Measurement of protein levels and coimmunoprecipitation. Protein levels were quantified in 6-month-old mice via Western blot. Briefly, cerebellar tissue was isolated manually, mechanically homogenized, solubilized in $2 \times$ Laemmli buffer, run in 4\%-20\% Tris/glycine gels, transferred onto nitrocellulose membranes, and labeled with an array of specific antibodies against multiple synaptic and cerebellar markers (see full list in attached Supplemental Table 3). Membranes were analyzed with a LI-COR Odyssey Infrared Imager. For coimmunoprecipitation experiments, $5 \times 10^{6}$ HEK293T cells were plated in $10-\mathrm{cm}$ dishes and transfected with plasmids containing mouse HA-tagged CaV2.1 cDNA (cytosolic C-terminal domain) and either rat Myctagged RIMBP-WT or RIMBP-MUT (p.Gly1808Ser). The variant was introduced using a Q5 Site-Directed Mutagenesis Kit (New England Biolabs). Cells were transfected with $6 \mu \mathrm{g}$ of each plasmid using Lipofectamine 2000 (Invitrogen) and harvested 24 hours after transfection. Cells were lysed on ice for 60 minutes in EBC lysis buffer (Boston BioProducts) containing cOmplete, Mini, EDTA-free Protease Inhibitor Cocktail (Roche, 11836170001) and subsequently centrifuged at 20,000 $\mathrm{g}$ for 10 minutes to remove cellular debris. Protein lysates were rotated overnight at $4^{\circ} \mathrm{C}$ with anti-Myc antibody (Cell Signaling Technology; 9B11). Twenty-five microliters of Dynabeads Protein $\mathrm{G}$ (Thermo Fisher Scientific) were then added to cell lysates and rotated at room temperature for 30 minutes. The samples were then washed 5 times, 5 minutes each, at room temperature with lysis buffer. Dynabeads were finally resuspended in $2 \times$ Laemmli buffer after the final wash and analyzed by standard SDS-PAGE and immunoblotting.

Statistics. Data in Figures 2, 3, and 4 are presented as the mean \pm SEM. Statistical significance was assessed by 1-way ANOVA (Figure 2, A and D, left; and Figure 4, D and G) with Tukey's post hoc analysis (Figure 4, D and G), or Student's 2-tailed $t$ test (Figure 2A bar graphs, $\mathrm{B}-\mathrm{D}$, right; Figure 3; Figure 4J). A $P$ value of less than 0.05 was considered significant, with levels of significance indicated in the figures as ${ }^{\star} P<0.05,{ }^{* *} P<0.01$, and ${ }^{* * *} P<0.001$; NS, not significant.

Study approval. The genetic studies were approved by the local ethics committees (family A - University College London Hospitals, London, UK; family B - University of Tartu, Tartu, Estonia; family C - Istanbul University, Istanbul, Turkey). Written informed consent for sample collection and subsequent analysis was obtained from all individuals or their guardians prior to inclusion in the study. All experiments involving mice were performed in accordance with Stanford and federal guidelines and were approved by the Stanford Institutional Animal Care and Use Committee and the animal and the animal ethics guidelines of the University of Heidelberg, Germany, under license T-52/18, and according to the regulations of Berlin authorities and animal welfare committee of the Charité-Universitätsmedizin Berlin, Germany under license no. 0220/09.

\section{Author contributions}

NEM, CA, and TCS conceived of and designed the study. CA, DK, NWW, TCS, CR, KO, EL, and TG acquired funding. NEM, MMB, JD, S Pajusalu, BA, PGL, CP, MS, GLC, JC, and GP conducted experiments. AT, A Pittman, JSS, BB, SW, TTW, A Papandreou, AS, RR, LKE, S Puusepp, KR, TT, HH, KPB, and MAK acquired data. CA, DK, NWW, TCS, CR, KO, and EL supervised the study. NEM and CA wrote the first draft of the manuscript. All authors contributed to revising the manuscript. NEM and MMB share first authorship because they contributed equally to data generation for this manuscript. NEM is listed first because of his role in the conception and coordination of the study and writing the first draft of the manuscript.

\section{Acknowledgments}

NEM is funded by a Parkinson's Foundation training grant. MS received a Heisenberg Fellowship from the German Research Council (DFG). This work is supported by NIH grant R37 NS096241 (to DK); the Chica and Heinz Schaller Foundation; NARSAD Young Investigator; DFG1158-SO2N (to CA); Estonian Research Council grants PUT355, PRG471, and PUTJD827 (to SP and KO); and a DAAD/ANID (57451854/62180003) fellowship (to JC). We thank Ülle Krikmann for providing some videos for patient B.

Address correspondence to: Niccolò E. Mencacci, Department of Neurology, Northwestern University Feinberg School of Medicine, Chicago, Illinois 60611, USA. Phone: 312.503.1781. Email: niccolo.mencacci@northwestern.edu. Or to: Claudio Acuna, Chica and Heinz Schaller Stiftung, Institute of Anatomy and Cell Biology, Heidelberg University, Im Neuenheimer Feld 307, Heidelberg 69120, Germany. Phone: 49.54.6221.8652. Email: acuna@ uni-heidelberg.de. 
1. Balint B, et al. Dystonia. Nat Rev Dis Primers. 2018;4(1):25.

2. Muller J, et al. The prevalence of primary dystonia in the general community. Neurology. 2002;59(6):941-943.

3. Albanese A, et al. Phenomenology and classification of dystonia: a consensus update. Mov Disord. 2013;28(7):863-873.

4. Jinnah HA, et al. The anatomical basis for dystonia: the motor network model. Tremor Other Hyperkinet Mov (N Y). 2017;7:506.

5. Schirinzi T, et al. Dystonia as a network disorder: a concept in evolution. Curr Opin Neurol. 2018;31(4):498-503.

6. Goodchild RE, et al. New genetic insights highlight 'old' ideas on motor dysfunction in dystonia. Trends Neurosci. 2013;36(12):717-725.

7. Tewari A, et al. It's not just the basal ganglia: cerebellum as a target for dystonia therapeutics. Mov Disord. 2017;32(11):1537-1545.

8. Lohmann K, Klein C. Update on the genetics of dystonia. Curr Neurol Neurosci Rep. 2017;17(3):26.

9. Jinnah HA, Sun YV. Dystonia genes and their biological pathways. Neurobiol Dis. 2019;129:159-168.

10. Calabresi P, et al. Hyperkinetic disorders and loss of synaptic downscaling. Nat Neurosci. 2016;19(7):868-875.

11. Waites CL, Garner CC. Presynaptic function in health and disease. Trends Neurosci. 2011;34(6):326-337.

12. Sudhof TC. The presynaptic active zone. Neuron. 2012;75(1):11-25.

13. Sudhof TC. Neurotransmitter release: the last millisecond in the life of a synaptic vesicle. $\mathrm{Neu}$ ron. 2013;80(3):675-690.

14. Hibino $\mathrm{H}$, et al. RIM binding proteins (RBPs) couple Rab3-interacting molecules (RIMs) to voltage-gated $\mathrm{Ca}(2+)$ channels. Neuron. 2002;34(3):411-423.

15. Liu KS, et al. RIM-binding protein, a central part of the active zone, is essential for neurotransmitter release. Science. 2011;334(6062):1565-1569.

16. Mittelstaedt T, Schoch S. Structure and evolution of RIM-BP genes: identification of a novel family member. Gene. 2007;403(1-2):70-79.

17. Davydova D, et al. Bassoon specifically controls presynaptic $\mathrm{P} / \mathrm{Q}-$ type $\mathrm{Ca}(2+)$ channels via RIM-binding protein. Neuron. 2014;82(1):181-194.

18. Acuna $\mathrm{C}$, et al. RIM-BPs mediate tight coupling of action potentials to $\mathrm{Ca}(2+)$-triggered neurotransmitter release. Neuron. 2015;87(6):1234-1247.

19. Acuna $C$, et al. How to make an active zone: unexpected universal functional redundancy between RIMs and RIM-BPs. Neuron. 2016;91(4):792-807.

20. Sobreira N, et al. GeneMatcher: a matching tool for connecting investigators with an interest in the same gene. Hum Mutat. 2015;36(10):928-930.

21. Puusepp S, et al. Effectiveness of whole exome sequencing in unsolved patients with a clinical suspicion of a mitochondrial disorder in Estonia. Mol Genet Metab Rep. 2018;15:80-89.

22. Scott EM, et al. Characterization of greater Middle
Eastern genetic variation for enhanced disease gene discovery. Nat Genet. 2016;48(9):1071-1076.

23. Lonsdale J, et al. The Genotype-Tissue Expression (GTEx) project. Nat Genet. 2013;45(6):580.

24. Lein ES, et al. Genome-wide atlas of gene expression in the adult mouse brain. Nature. 2007;445(7124):168-176.

25. Schwenk F, et al. A cre-transgenic mouse strain for the ubiquitous deletion of loxP-flanked gene segments including deletion in germ cells. Nucleic Acids Res. 1995;23(24):5080-5081.

26. Pelosi A, et al. Heterozygous gnal mice are a novel animal model with which to study dystonia pathophysiology. J Neurosci. 2017;37(26):6253-6267.

27. Luo F, et al. Efficient stimulus-secretion coupling at ribbon synapses requires RIM-binding protein tethering of L-type $\mathrm{Ca}(2+)$ channels. Proc Natl Acad Sci U S A. 2017;114(38):E8081-E8090.

28. Grauel MK, et al. RIM-binding protein 2 regulates release probability by fine-tuning calcium channel localization at murine hippocampal synapses. Proc Natl Acad Sci U S A. 2016;113(41):11615-11620.

29. Bekkers JM, Stevens CF. Excitatory and inhibitory autaptic currents in isolated hippocampal neurons maintained in cell culture. Proc Natl Acad Sci U S A. 1991;88(17):7834-7838.

30. Arancillo M, et al. Titration of Syntaxin1 in mammalian synapses reveals multiple roles in vesicle docking, priming, and release probability. J Neurosci. 2013;33(42):16698-16714.

31. Le Ber I, et al. Predominant dystonia with marked cerebellar atrophy: a rare phenotype in familial dystonia. Neurology. 2006;67(10):1769-1773.

32. Richter F, Richter A. Genetic animal models of dystonia: common features and diversities. Prog Neurobiol. 2014;121:91-113.

33. Calderon DP, et al. The neural substrates of rapidonset dystonia-parkinsonism. Nat Neurosci. 2011;14(3):357-365.

34. Fremont R, et al. Abnormal high-frequency burst firing of cerebellar neurons in rapidonset dystonia-parkinsonism. J Neurosci. 2014;34(35):11723-11732.

35. Fremont R, et al. A role for cerebellum in the hereditary dystonia DYT1. Elife. 2017;6:e22775.

36. Fremont R, et al. Aberrant Purkinje cell activity is the cause of dystonia in a shRNA-based mouse model of rapid onset dystonia-parkinsonism. Neurobiol Dis. 2015;82:200-212.

37. Washburn S, et al. Acute cerebellar knockdown of Sgce reproduces salient features of myoclonus-dystonia (DYT11) in mice. Elife. 2019;8:e52101.

38. Chen $\mathrm{CH}$, et al. Short latency cerebellar modulation of the basal ganglia. Nat Neurosci. 2014;17(12):1767-1775.

39. Eskow Jaunarajs KL, et al. Striatal cholinergic dysfunction as a unifying theme in the pathophysiology of dystonia. Prog Neurobiol. 2015;127-128:91-107.

40. White JJ, Sillitoe RV. Genetic silencing of olivocerebellar synapses causes dystonia-like behaviour in mice. Nat Commun. 2017;8:14912.
41. Brockmann MM, et al. RIM-BP2 primes synaptic vesicles via recruitment of Munc13-1 at hippocampal mossy fiber synapses. Elife. 2019;8:e43243.

42. Sclip A, et al. RIM-binding proteins recruit BK-channels to presynaptic release sites adjacent to voltage-gated $\mathrm{Ca}(2+)$-channels. EMBO J. 2018;37(16):e98637.

43. Alvina K, Khodakhah K. KCa channels as therapeutic targets in episodic ataxia type-2. J Neurosci. 2010;30(21):7249-7257.

44. Spacey SD, et al. Two novel CACNA1A gene mutations associated with episodic ataxia type 2 and interictal dystonia. Arch Neurol. 2005;62(2):314-316.

45. Du W, et al. Calcium-sensitive potassium channelopathy in human epilepsy and paroxysmal movement disorder. Nat Genet. 2005;37(7):733-738.

46. Zhang ZB, et al. De novo KCNMA1 mutations in children with early-onset paroxysmal dyskinesia and developmental delay. Mov Disord. 2015;30(9):1290-1292.

47. Reinson K, et al. Biallelic CACNA1A mutations cause early onset epileptic encephalopathy with progressive cerebral, cerebellar, and optic nerve atrophy. Am JMed Genet A. 2016;170(8):2173-2176.

48. Tabarki B, et al. Homozygous KCNMA1 mutation as a cause of cerebellar atrophy, developmental delay and seizures. Hum Genet. 2016;135(11):1295-1298.

49. Gorman KM, et al. Bi-allelic loss-of-function CACNA1B mutations in progressive epilepsy-dyskinesia. Am JHum Genet. 2019;104(5):948-956.

50. Shen Y, et al. Protein mutated in paroxysmal dyskinesia interacts with the active zone protein RIM and suppresses synaptic vesicle exocytosis. Proc Natl Acad Sci U S A. 2015;112(10):2935-2941.

51. Lipstein N, et al. Synaptic UNC13A protein variant causes increased neurotransmission and dyskinetic movement disorder. JClin Invest. 2017;127(3):1005-1018.

52. Bucan M, et al. Genome-wide analyses of exonic copy number variants in a family-based study point to novel autism susceptibility genes. PLoS Genet. 2009;5(6):1000536.

53. Purcell S, et al. PLINK: a tool set for wholegenome association and population-based linkage analyses. Am JHum Genet. 2007;81(3):559-575.

54. Magi A, et al. H3M2: detection of runs of homozygosity from whole-exome sequencing data. Bioinformatics. 2014;30(20):2852-2859.

55. Carecchio $\mathrm{M}$, et al. Frequency and phenotypic spectrum of KMT2B dystonia in childhood: a single-center cohort study. Mov Disord. 2019;34(10):1516-1527.

56. Pajusalu S, et al. Novel homozygous mutation in KPTN gene causing a familial intellectual disability-macrocephaly syndrome. Am JMed Genet A. 2015;167A(8):1913-1915.

57. Atasu B, et al. HPCA confirmed as a genetic cause of DYT2-like dystonia phenotype. Mov Disord. 2018;33(8):1354-1358. 